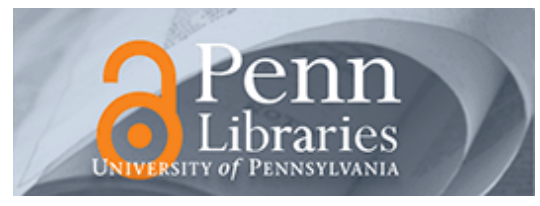

University of Pennsylvania

ScholarlyCommons

Statistics Papers

Wharton Faculty Research

1994

\title{
Characterization of Probability Law by Absolute Moments of Its Partial Sums
}

\author{
M. Braverman \\ C. Mallows \\ Larry A. Shepp \\ University of Pennsylvania
}

Follow this and additional works at: https://repository.upenn.edu/statistics_papers

Part of the Probability Commons

\section{Recommended Citation}

Braverman, M., Mallows, C., \& Shepp, L. A. (1994). Characterization of Probability Law by Absolute Moments of Its Partial Sums. Theory of Probability \& Its Applications, 40 (2), 238-249. http://dx.doi.org/ $10.1137 / 1140027$

This paper is posted at ScholarlyCommons. https://repository.upenn.edu/statistics_papers/407

For more information, please contact repository@pobox.upenn.edu. 


\title{
Characterization of Probability Law by Absolute Moments of Its Partial Sums
}

\author{
Abstract \\ If $S_{n}=X_{1}+\ldots+X_{n}$, where $X_{i}$ are independent and identically distributed (i.i.d.) standard normal, then \\ $E\left|S_{n}\right| \equiv \sqrt{ } 2 n / \pi, n \geqq 0$. We show that no other symmetric law has exactly these "moments"; the general \\ case remains (stubbornly) open. If $X$ is standard two-sided exponential, then $E\left|S_{n}\right|=2 n 2^{-2 n(2 n / n)}$. We show \\ the latter moments are obtained exactly for all $n$ also for $X_{i} \sim B(2 ; 0.5)$, the sum of two standard $( \pm$ \\ 1-valued) Bernoulli's as well as for many other laws including unsymmetrical ones: $X_{i} \sim G-1$, where $G$ is \\ geometric with mean 1 , is one example. \\ Our interest in this delicate nonlinear inverse problem (which was initiated by Klebanov, cf. [12]) of \\ inverting the moments to recover the law was also drawn by the fact that it gives a way to study positive \\ definite functions through the formula $\mathrm{E}\left|\mathrm{S}_{n}\right|=(2 / \pi) \int 0^{\infty} \operatorname{Re}\left(1-\varphi^{\mathrm{n}}(1 / \mathrm{u})\right) \mathrm{d} u, n \geqq 0$, expressing $\mathrm{E}\left|\mathrm{S}_{n}\right|$ in \\ terms of the moments of $\varphi$, where $\varphi$ is the characteristic function of $X, \varphi(u)=\operatorname{Eexp}(i u X)$. We show that if \\ for some $b>0, \psi_{b}(u)=\varphi(b \tan (u / b))$ is a positive definite function then the distributions corresponding \\ to $\varphi$ and $\psi_{b}$ have the same $E\left|S_{n}\right|$ moments for all $n$. \\ We show that if $X$ is Bernoulli with zero mean and values \pm 1 then the moments characterize the \\ distribution uniquely even among nonsymmetric laws. In general however we expect that the moments do \\ not characterize the law, and this may well be the only nontrivial case of uniqueness. \\ We extend some of our results to the case of $p$ th moments, $p$ different from an even integer.

\section{Keywords} \\ independent identically distributed random variables, absolute moments of partial sums, induced \\ measure of characteristic function, symmetric and unsymmetric laws, positively defined function

\section{Disciplines} \\ Probability | Statistics and Probability
}




\title{
CHARACTERIZATION OF A PROBABILITY LAW BY ABSOLUTE MOMENTS OF ITS PARTIAL SUMS*
}

\author{
M. BRAVERMAN ${ }^{\dagger}$, C. MALLOWS $\ddagger$, AND L. SHEPP $\ddagger$
}

\begin{abstract}
If $S_{n}=X_{1}+\cdots+X_{n}$, where $X_{i}$ are independent and identically distributed (i.i.d.) standard normal, then $\mathbf{E}\left|S_{n}\right| \equiv \sqrt{2 n / \pi}, n \geqq 0$. We show that no other symmetric law has exactly these "moments"; the general case remains (stubbornly) open. If $X$ is standard two-sided exponential, then $\mathbf{E}\left|S_{n}\right|=2 n 2^{-2 n}\left(\begin{array}{c}2 n \\ n\end{array}\right), n \geqq 0$. We show the latter moments are obtained exactly for all $n$ also for $X_{i} \sim B(2 ; 0.5)$, the sum of two standard ( \pm 1 -valued) Bernoulli's as well as for many other laws including unsymmetrical ones: $X_{i} \sim G-1$, where $G$ is geometric with mean 1 , is one example.

Our interest in this delicate nonlinear inverse problem (which was initiated by Klebanov, cf. [12]) of inverting the moments to recover the law was also drawn by the fact that it gives a way to study positive definite functions through the formula $\mathbf{E}\left|S_{n}\right|=(2 / \pi) \int_{0}^{\infty} \operatorname{Re}\left(1-\varphi^{n}(1 / u)\right) d u, n \geqq 0$, expressing $\mathbf{E}\left|S_{n}\right|$ in terms of the moments of $\varphi$, where $\varphi$ is the characteristic function of $X, \varphi(u)=$ $\mathbf{E} \exp (i u X)$. We show that if for some $b>0, \psi_{b}(u)=\varphi(b \tan (u / b))$ is a positive definite function then the distributions corresponding to $\varphi$ and $\psi_{b}$ have the same $\mathbf{E}\left|S_{n}\right|$ moments for all $n$.

We show that if $X$ is Bernoulli with zero mean and values \pm 1 then the moments characterize the distribution uniquely even among nonsymmetric laws. In general however we expect that the moments do not characterize the law, and this may well be the only nontrivial case of uniqueness.

We extend some of our results to the case of $p$ th moments, $p$ different from an even integer.
\end{abstract}

Key words. independent identically distributed random variables, absolute moments of partial sums, induced measure of characteristic function, symmetric and unsymmetric laws, positively defined function

1. Introduction. Let $\mathcal{A}$ be a set of probability distributions with finite $p$ th moment and $\left\{X_{k}\right\}$ independent identically distributed random variables (i.i.d. r.v.'s) with a common distribution $F \in \mathcal{A}$. We say that $F$ is determined in $\mathcal{A}$ by the sequence

$$
a_{n}=\mathbf{E}\left|\sum_{k=1}^{n} X_{k}\right|^{p} \quad(n \in \mathbf{N}),
$$

if whenever for all $n \in \mathbf{N}$,

$$
\mathbf{E}\left|\sum_{k=1}^{n} X_{k}\right|^{p} \equiv \mathbf{E}\left|\sum_{k=1}^{n} Y_{k}\right|^{p},
$$

where $\left\{Y_{k}\right\}$ are i.i.d. r.v.'s with distribution $G \in \mathcal{A}$, then $G \equiv F$. We say $F$ is determined (or is determined in general) if $\mathcal{A}$ is the set of all distributions.

M. Neupokoeva [12] proved that if $0<p<2$ and $\mathcal{A}$ is the class of symmetric distributions with characteristic function $\varphi$ decreasing on $[0, \infty)$ then each $F \in \mathcal{A}$ is determined in $\mathcal{A}$. Braverman [1] then showed that Neupokoeva's conditions can be replaced by the following ones: $\varphi(z)$ analytic near $z=0$; $\lim \sup \varphi(z)<1$ as $z \rightarrow \infty$, i.e., Cramer's condition, $C$ (for $p>0$ not an even integer), again assuming $\mathcal{A}$ to be the class of symmetrical laws. But what if the two hypotheses on $\varphi$, (a) $\varphi$ is real and (b) $\varphi$ satisfies $C$, are removed? We show that if condition (b) is removed then there may indeed be cases when for two symmetric distributions (one with a periodic

\footnotetext{
*Received by the editors July 29, 1994.

$\dagger_{\text {Math. Institute, Khabarovsk, Russia. }}$

‡AT\&T Bell Laboratories, Murray Hill, NJ 07974.
} 
characteristic function) (1.1) holds. A simple example is when $X$ and $Y$ have the respective characteristic functions

$$
\varphi(z)=\frac{1}{1+z^{2}} \quad \text { and } \quad \psi(z)=\cos ^{2} z,
$$

corresponding to the two-sided exponential, $\mathbf{P}\left\{X_{k} \in d x\right\}=\frac{1}{2} e^{-|x|} d x$, and $B_{2}$, the symmetric Bernoulli distribution of order $2, \mathbf{P}\left\{Y_{k}=2\right\}=\mathbf{P}\left\{Y_{k}=-2\right\}=\frac{1}{4}, \mathbf{P}\left\{Y_{k}=\right.$ $0\}=\frac{1}{2}$. We have that (1.1) holds (for $p=1$ ), and this is the first known example of nonuniqueness. However, we believe the situation is that, except for the case of symmetric one- and two-valued random variables $X$, unique characterization never holds. We are very far from proving this however.

We show that there is no such (symmetric) partner for the standard normal law, i.e., the standard normal is determined in the set, $\mathcal{S}$, of symmetric distributions (in distinction to the two-sided exponential which is not determined in $\mathcal{S}$ ). Whether or not the normal law is determined in general remains open and is a rather interesting question, as we shall see.

We expect that "most" distributions in $\mathcal{S}_{1}$ with a characteristic function which is decreasing, or which satisfies condition $C$, are not determined in $\mathcal{S}_{1}$. This is because, as we shall show in $\S 2$, if $\varphi(z)$ is any characteristic function (not necessarily real) and if

$$
\psi(z)=\psi_{b}(z) \triangleq \varphi\left(b \tan \frac{z}{b}\right)
$$

for some fixed $b>0$, is also positive definite, i.e., $\psi(z)$ is a characteristic function, then the distributions with characteristic function $\varphi$ and $\psi$ have the same sequence (1.1). As $b \rightarrow \infty, \psi_{b}(z) \rightarrow \varphi(z)$ so one would "expect" (although it can fail) that $\psi_{b}$ is positive definite for sufficiently large $b$. Note the example in (1.2) has $b=1$ since $1 /\left(1+\tan ^{2} z\right)=\cos ^{2} z$ but we shall see that $\psi_{b}(z)$ is actually positive definite for all $b \geqq 1$ in this case.

We prove in $\S 2$ that (for $p=1$ ) the standard symmetric Bernoulli distribution with $\mathbf{P}\left\{X_{k}=1\right\}=\mathbf{P}\left\{X_{k}=-1\right\}=\frac{1}{2}$, is determined in class $\mathcal{L}_{1}$; this is the only example of a distribution we know that is determined without any restrictions. We show that a nonsymmetric two-point zero mean law is also determined in $\mathcal{L}_{1}$ (except the trivial case $-X_{k}$ ).

The method of characteristic functions, introduced by Neupokoeva, becomes hard to use in the asymmetric case. Clearly if $X_{1}$ is stable with index $p<\alpha<2$ and arbitrary skewness, and $Y_{1}$ is a suitable multiple of a standard symmetric stable with the same $\alpha$, then $\mathbf{E}\left|X_{1}+\cdots+X_{n}\right|^{p}=n^{p / \alpha} \mathbf{E}\left|X_{1}\right|=\mathbf{E}\left|Y_{1}+\cdots+Y_{n}\right|^{p}$. Another example (with $p=1$ ) of a symmetric and an asymmetric distribution having the same sum-moments is given by $B(2 ; 0.5)$ and $G-1$, as seen in $(2.15)-(2.16)$. On the other hand, one of us would guess that the class of unsymmetric laws is rich enough to duplicate the sequence of moments in (1.1) for "most" symmetric laws, e.g., $N(0,1)$ (the standard Bernoulli case is an exception (maybe the only one?) and is a boundary case), but we have no idea how to prove anything like this, and another of us would guess that $B(3 ; 0.5)$ would provide another example of uniqueness.

In $\S 2$, we give proofs of our results for the case $p=1$; insofar as this is possible we extend this result to $p \neq 1$ in $\S 3$ except of course $p$ cannot be an even integer, since the values of $\mathbf{E}\left|X_{1}+\cdots+X_{n}\right|^{p}$ clearly cannot determine the distribution since any law with the same first $p$ integer moments will give the same values. 
Another reason for interest in this class of problems, besides the study of characteristic functions and distributions, is that the problem of recovering the distribution from the moments of partial sums is a rather delicate inverse problem. Indeed, for every distribution with zero mean and unit variance, the asymptotics of sequence $\mathbf{E}\left|\sum_{k=1}^{n} X_{k}\right|^{p}$ are the same, viz. $\sqrt{2 n / \pi}$ (for $p=1$ ), as a consequence [2] of the central limit theorem. This result of [2] motivated our problem: if the latter moments hold not only asymptotically but exactly for all $n$, does this characterize $N(0,1)$ ? The spirit of the problem is close to that of characterizing a distribution from the mean values of the order statistics [8], [11].

2. Proofs for the case $p=1$. The case $p=1$ is slightly simpler so we restrict attention for clarity to this case here; the general case is treated in $\S 3$.

The reason that characteristic functions enter is because of the following formula, valid for any r.v. $X$ and its characteristic function, $\varphi$ :

$$
\mathbf{E}|X|=\frac{2}{\pi} \int_{0}^{\infty} \frac{1-\operatorname{Re} \varphi(t)}{t^{2}} d t=P . V \cdot \frac{1}{\pi} \int_{-\infty}^{\infty} \frac{1-\varphi(u)}{u^{2}} d u .
$$

To prove (2.1) merely note that for any real $a$,

$$
|a|=\frac{2}{\pi} \int_{0}^{\infty} \frac{1-\cos a t}{t^{2}} d t .
$$

Set $a=X$ and take expectations using Fubini's theorem, and note that $\varphi(-u)=\overline{\varphi(u)}$ so the last integral in (2.1) takes the real part. If $\mathbf{E} X=0$ and $\mathbf{E} X^{2}<\infty$ then $(1-\varphi(u)) / u^{2} \in L^{1}$ and the principal value in (2.1) can be dropped.

Applying (2.1) to $S_{n}=X_{1}+\cdots+X_{n}$ we obtain

$$
\mathbf{E}\left|S_{n}\right|=\frac{2}{\pi} \int_{0}^{\infty} \frac{1-\operatorname{Re} \varphi^{n}(t)}{t^{2}} d t=\frac{2}{\pi} \int_{0}^{\infty}\left(1-\operatorname{Re} \varphi^{n}\left(\frac{1}{u}\right)\right) d u
$$

after substituting $t=1 / u$.

If now $\varphi$ is real then the real part in (2.3) involves just $\varphi(1 / u)$ itself and so the ordinary moments of $\varphi(1 / u)$ enter in (2.3). We write for a real characteristic function $\varphi$ and $A \subset[-1,1]$

$$
H_{\varphi}(A)=\operatorname{Leb}\left\{0<u<\infty: \varphi\left(\frac{1}{u}\right) \in A\right\}
$$

where Leb is Lebesgue measure. Note that $H_{\varphi}(A)<\infty$ if $A \subset[-1,1-\varepsilon]$ for any $\varepsilon>0$ but $H_{\varphi}[-1,1]=\operatorname{Leb}([0, \infty])=\infty$. We may call $H$ the induced measure of the characteristic function of $1 / u$, and we obtain from (2.3), so long as $\varphi$ is real,

$$
\mathbf{E}\left|S_{n}\right|=\frac{2}{\pi} \int_{-1}^{1}\left(1-z^{n}\right) H_{\varphi}(d z), \quad n \geqq 1 .
$$

It is now obvious that if two characteristic functions have the same induced measure $H$ then the pseudomoments, $\mathbf{E}\left|S_{n}\right|$, will be the same. For real characteristic functions the converse is also valid (shown by Neupokoeva [12] and Braverman [1]). Thus real $\varphi$ and $\psi$ have the same moments (2.5) if and only if $H_{\varphi} \equiv H_{\psi}$. Motivated by this we try to find a periodic counterexample and set for fixed $b>0$

$$
\psi(z)=\psi_{b}(z)=\varphi\left(b \tan \frac{z}{b}\right) .
$$


If it turns out that $\psi=\psi_{b}$ is positive definite, then its induced measure $H_{\psi}$ will be the same as that of $\varphi$. To verify this, it is easiest to argue directly from (2.1) using $(-\infty, \infty)$ instead of $(0, \infty)$ for convenience since $\varphi$ is even. We have

$$
\frac{1}{\pi} \int_{-\infty}^{\infty} \frac{1-\psi_{b}^{n}(t)}{t^{2}} d t=\frac{1}{\pi} \int_{-\infty}^{\infty} \frac{1-\varphi^{n}(b \tan (t / b))}{t^{2}} d t .
$$

Taking into account that $\psi_{b}$ has period $b \pi$ we may write $(2.7)$ in the form

$$
\frac{1}{\pi} \int_{-\pi b / 2}^{\pi b / 2}\left(1-\varphi^{n}\left(b \tan \frac{t}{b}\right)\right) \sum_{k=-\infty}^{\infty} \frac{1}{(k \pi b+t)^{2}} d t .
$$

Applying the well-known formula

$$
\sum_{k=-\infty}^{\infty} \frac{1}{(k \pi b+t)^{2}}=\frac{1}{b^{2} \sin ^{2}(t / b)}
$$

and making the change of variables $b \tan (t / b)=u, \sec ^{2}(t / b) d t=d u$, we get

$$
\frac{1}{\pi} \int_{-\infty}^{\infty}\left(1-\varphi^{n}(u)\right) \frac{d u}{\sec ^{2}(t / b)} \frac{1}{b^{2} \sin ^{2}(t / b)}=\frac{2}{\pi} \int_{0}^{\infty} \frac{1-\varphi^{n}(u)}{u^{2}} d u
$$

since $u^{2}=b^{2} \tan ^{2}(t / b)$, so that the values of (2.3) are the same for both $\varphi$ and $\psi_{b}$. If $\varphi$ is real (note (2.6)-(2.10) are valid even for complex $\varphi$ ) then it follows from (2.5)-(2.10) that the induced measures $H_{\varphi}$ and $H_{\psi}$ are the same and so the respective moments $\mathbf{E}\left|S_{n}\right|$ are the same (of course $\psi$ must be a characteristic function).

Indeed it was the attempt to make the induced measures the same for a decreasing $\varphi$ and a periodic $\psi$ that led us to the transformation (2.6).

Note that Cramer condition $\mathrm{C}$ fails to hold for periodic functions $\psi_{b}$ so there is no contradiction with the theorem of Braverman [11].

If now we take

$$
\varphi(z)=\frac{1}{1+z^{2}}=\int_{-\infty}^{\infty} \frac{1}{2} \exp (-|x|) \exp (i x z) d x
$$

and we take $b=1$ in $(2.6)$, then $\psi(z)=\varphi(\tan z)=\cos ^{2} z$ is indeed positive definite since $\psi(z)=\mathbf{E} \exp (i Y z)$, where $Y=B\left(2, \frac{1}{2}\right)$ is the sum of two \pm 1 valued Bernoulli variables which gives (1.2). Actually, we have

$$
\psi_{b}(b z)=\frac{1}{1+b^{2} \tan ^{2} z}=\frac{\cos ^{2} z}{b^{2}-\left(b^{2}-1\right) \cos ^{2} z}
$$

is positive definite for any $b \geqq 1$, as is easily checked by expanding out the right side of (2.11) in powers of $\cos ^{2} z$ since we get all positive coefficients. For $0<b<1, \psi_{b}(b z)$ is not positive definite, as can be seen by calculating

$$
\mathbf{P}\left\{X_{b}=2\right\}=\frac{1}{\pi} \int_{-\pi}^{\pi}\left(1+b^{2} \tan ^{2} z\right)^{-1} \cos 2 z d z=2 b \frac{b-1}{(b+1)^{3}}<0 .
$$

In general since $\psi_{b}(z)=\varphi(b \tan (z / b)) \longrightarrow \varphi(z)$ as $b \rightarrow \infty$, it is reasonable to expect that for sufficiently large $b, \psi_{b}(z)$ will indeed be positive definite since it converges to a positive definite limit. Although is probably true for "most" $\varphi$, it is not true for 
the standard normal since $\psi(z)=\exp \left\{-\left(b^{2} / 2\right) \tan ^{2} z\right\}$ is not positive definite for any $b$ because of the theorem [10, Chap. 7] that if a characteristic function, $\psi$, is analytic in a circle $|z|<R$ then it is analytic in the strip $|\operatorname{Im} z|<R$, but $\psi(z)$ has an essential singularity at $z=\pi / 2$; note that in (2.11) the value $z=\pi / 2$ merely becomes a zero of $\psi$. Further examples of this type can be given if we take $\varphi_{\alpha}(z)$ to be the characteristic function whose density function is $c \exp \left(-|z|^{\alpha}\right)$. Function $\varphi_{\alpha}(z)$ is analytic for every choice of $1<\alpha \leqq 2$. We suspect that $\exp \left(-b|\tan z|^{\alpha}\right)$ is positive definite for large $b$ for $1<\alpha<2$ which would show that there are distributions with the same sum-moments as the symmetric stable laws, but we can't prove this.

Next we show that each of $\varphi_{\alpha}(z)$ is uniquely determined by its pseudomoments $\mathbf{E}\left|S_{n}\right|, n \geqq 1$, among the class of all symmetric laws. Note that if the characteristic function $\varphi$ is even and decreasing on $(0, \infty)$, then $\varphi(t)=0$ for all $t$. Really, in the opposite case $\varphi(t) \rightarrow c<0$ as $t \rightarrow \infty$, which implies

$$
\lim _{T \rightarrow \infty} \frac{1}{2 T} \int_{-T}^{T} \varphi(t) d t=c<0 .
$$

But the last limit cannot be negative since it equals the jump at zero of the respective distribution function [10, p. 54].

THEOREM 2.1. If $\varphi(z)$ is analytic and if $\varphi(z)$ decreases to zero as $z \rightarrow \infty$ in such a way that $H_{\varphi}$ has a continuous Radon-Nicodym derivative in $(0,1)$ in $z$ on $(0, \infty]$, and if $\psi=\psi_{b}$ in (2.6) is not positive definite for any $b>0$, then the law of $\varphi$ is determined by the sum-moments among the class of all symmetric distributions.

Proof. We have seen that $H_{\psi} \equiv H_{\varphi}$ since the equality of the pseudomoments implies that the induced measures are the same if $\varphi$ and $\psi$ are both real. If $\psi(z)$ is also monotonic in $z$ on $[0, \infty)$ then $(2.4)$ implies easily that $\psi(1 / u) \equiv \varphi(1 / u)$ and so $\varphi=\psi$ and the laws are identical. Consider now when $\psi$ is not decreasing on $[0, \infty)$. Show that if $\varphi \in C^{2}$, then $\psi \in C^{2}$. Indeed, $\mathbf{E} X_{k}^{2}<\infty$. From [2] it follows that the latter relation is equivalent to $\mathbf{E}\left|S_{n}\right|=O\left(n^{1 / 2}\right)$. In view of the equality of moments of respective sums, we have $\mathbf{E} Y^{2}<\infty$ which gives the statement we need.

Suppose there is some point $a \in(0, \infty)$ for which $0<\psi(1 / a)<1$ and $\psi^{\prime}(1 / a)=0$. Then $H_{\psi}(d A)$ has an infinite Radon-Nicodym derivative at the point $b=\psi(1 / a) \in$ $(0,1)$. This is because in $(2.4)$

$$
\begin{aligned}
\frac{1}{\delta} \operatorname{Leb}\left\{0<u<\infty:\left|\psi\left(\frac{1}{u}\right)-\psi\left(\frac{1}{a}\right)\right|<\delta\right\} & \geqq \frac{1}{\delta} \operatorname{Leb}\left\{u:|u-a|<\sqrt{\frac{\delta}{|2 c|}}\right\} \\
& =2 \sqrt{\frac{1}{|2 c| \delta}}
\end{aligned}
$$

because for $u$ near $a, \psi(1 / u)=b+c(u-a)^{2}+o\left((u-a)^{2}\right)$, where $c=\frac{1}{2} \psi^{\prime \prime}(1 / a) a^{-4}$, since $\psi^{\prime}(1 / a)=0$. Hence the right-hand side tends to $\infty$ as $\delta \rightarrow 0$. But $H_{\varphi}$ and $H_{\psi}$ must have the same Radon-Nicodym derivative because $H_{\varphi}=H_{\psi}$. So if $a \in(0, \infty)$ and $\psi^{\prime}(1 / a)=0$ then $\psi(1 / a)=0$ or 1 , the only points where $H_{\varphi}$ can have an infinite Radon-Nicodym derivative.

We assumed that $\psi$ is not decreasing on $[0, \infty)$. But since its first derivative vanished at zero, the second derivative is negative, then $\psi(u)$ decreases at zero from the right. Then there exists $a>0$ point $a$ such that $\psi^{\prime}(a)=0$ and $\psi^{\prime}(t)<0$ for $0<t<a$. This implies that $\psi(a)=0$ and two cases are possible: (1) there exists 
a point $c>a$ such that $\psi(c)=1$ and $\psi(t)$ increases on $(a, c) ;(2)$ the function $\psi$ increases on $(a, \infty)$.

In the first case $\psi$ must be periodic and from $H_{\varphi}=H_{\psi}$ it follows that (2.6) holds , i.e., $\psi(z) \equiv \psi_{b}(z)$ for some $b>0$. But we have assumed in Theorem 2.1 that $\psi_{b}(t)$ is not positive definite. So the case (1) is impossible. Let assumption (2) hold. Then $\psi(t)$ tends to some limit $d \leqq 1$ as $t \rightarrow \infty$. Show that $d \neq 1$. Note that the determinant $\psi_{i j}=\psi\left(t_{i}-t_{j}\right)$, where $\left(t_{1}, t_{2}, t_{3}\right)=(0, a, t)$, must be positive. But $\psi_{11}=\psi_{22}=\psi_{33}=1, \psi_{12}=\psi_{21}=0$, and $\psi_{i j} \sim 1$ for other values of $i$ and $j$. So the determinant tends to -1 as $t \rightarrow \infty$.

Applying the known inequality [9, p. 208]

$$
|\psi(t+h)-\psi(t)|^{2} \leqq 2(1-\psi(h)),
$$

which true for all $t$ and $h$, we can get another proof. Supposing $h \rightarrow \infty$ we obtain $\psi(t)=1$ for all $t$, which contradicts the previous relations.

So $d<1$. (2.4), relation $H_{\varphi}=H_{\psi}$, and the fact that $\varphi$ and $\psi$ decrease near zero imply that $\varphi(1 / u)=\psi(1 / u)$ for $u$ sufficiently large, i.e., $\varphi(t)=\psi(t)$ near zero. Since $\varphi$ is analytic near zero, then the last equality holds for all $t$ (see [6, Chap. 1]). The theorem is proved.

It is easy to see that $\varphi(z)=\exp \left(-z^{2} / 2\right)$ satisfies the hypothesis (the same holds for $\varphi_{\alpha}(z)$ as defined above for $\left.1<\alpha<2\right)$

$$
\begin{aligned}
\frac{d}{d x} H_{\varphi}((0, x)) & =\frac{d}{d x} \operatorname{Leb}\left\{e^{-\left(1 / 2 u^{2}\right)} \leqq x\right\}=\frac{d}{d x} \frac{1}{\sqrt{2 \log 1 / x}} \\
& =\frac{1}{\sqrt{8} x(\log 1 / x)^{3 / 2}},
\end{aligned}
$$

which is finite and continuous for $0<x<1$ as required. The fact that respective $\psi_{b}$ are not positive definite functions was stated above. One can show (we omit the proof) that functions $\varphi_{\alpha}(z)$ defined above satisfied the theorem's assumptions for $1<\alpha<2$.

In the unsymmetrical case, there is still a measure $H_{\varphi}$ induced as in (2.5) but it lives on the unit disk, $D$, rather than on the interval $[-1,1]$. However, for $\varphi$ real a measure $\mu$ on the disk is not determined from the values of

$$
\int_{D}\left(1-z^{n}\right) d \mu(z)
$$

since if $\mu$ is a uniform measure on a circle of radius $r$ which is contained in $\{|z|<1\}$ then (2.14) is independent of $r$ because $1-z^{n}$ is a harmonic function on the disk $D$. Of course such a measure $\mu$ is not an $H_{\varphi}$ for a characteristic function $\varphi$. Thus the complex or unsymmetrical case is very different from the case of real $\varphi$, where the moments in (2.5) uniquely determined $H_{\varphi}$. We next give an example of a pair of distributions with the same pseudomoments with one symmetrical and one unsymmetrical. It follows that in general the moments (2.14) do not determine $\mu$ even if $\mu$ is a measure induced by a characteristic function.

We have seen in (2.11) that all $\psi_{b}(z)$ for $b \geqq 1$ are characteristic functions, with $H_{\psi_{b}}$ all the same. Now we will show that there are unsymmetric laws having complex characteristic functions, $\varphi$, with the same moments, hence the measure $H_{\varphi}$ is not supported on $[-1,1]$ and so the moments $(2.14)$ do not determine $\mu$ even for characteristic functions if we can find such a law. We gave another example below (1.3) using stable laws for $\alpha>1$. 
First we show by direct calculation that the common moments for all $\psi_{b}, b \geqq 1$, in (2.11), which are easily seen to be

$$
\mathbf{E}\left|S_{n}\right|=2 n 2^{-2 n}\left(\begin{array}{c}
2 n \\
n
\end{array}\right), \quad n \geqq 1,
$$

are also obtained for the unsymmetrical law $G-1$ where $G$ is geometric, with $\mathbf{P}\{G=$ $j\}=2^{-(j+1)}, j=0,1,2, \ldots$. The moments for $G-1$ are

$$
\sum_{k=0}^{\infty} \mathbf{P}\left\{G_{1}+\cdots+G_{n}=k\right\}|k-n|=2 \sum_{k=n}^{\infty} \mathbf{P}\left\{G_{1}+\cdots+G_{n}=k\right\}(k-n)
$$

since $\mathbf{E}\left(G_{1}+\cdots+G_{n}-n\right)=0$ so the sum on $0 \leqq k \leqq n$ is the same as that on $n \leqq k<\infty$. But we have explicitly that

$$
\mathbf{P}\left\{G_{1}+\cdots+G_{n}=k\right\}=\left(\begin{array}{c}
n+k-1 \\
n-1
\end{array}\right) 2^{-(n+k)},
$$

so that the sum in (2.16) can be written as the telescoping sum

$$
\begin{aligned}
& 2^{-n} \sum_{k=n}^{\infty}\left[\left(\begin{array}{c}
n+k-1 \\
n-1
\end{array}\right) 2^{-(k-1)} k-\left(\begin{array}{c}
n+k \\
n-1
\end{array}\right) 2^{-k}(k+1)\right] \\
& \quad=2^{-n}\left(\begin{array}{c}
2 n-1 \\
n-1
\end{array}\right) 2^{-(n-1)} n
\end{aligned}
$$

which is the same as (2.15). So the measure $H_{\varphi}$ induced by $\varphi(z)=\mathbf{E} e^{i(G-1) z}=$ $e^{-i z} /\left(2-e^{i z}\right)$, which is not concentrated on $[-1,1]$, is certainly different from the measures $H_{\psi_{b}}$ (which are all the same), which are concentrated on $[-1,1]$. The trick used in (2.8) provides us with a convenient formula whenever the random variable $X$ has lattice support. Suppose the characteristic function of $X$ is

$$
\varphi(t)=\sum_{-\infty}^{\infty} p_{j} e^{i j a t}=\sum_{-\infty}^{\infty} p_{j} z^{j}=\rho(z) \quad \text { say }
$$

where $z=e^{i a t}$. Then

$$
\mathbf{E}\left|S_{n}\right|=-\frac{a}{\pi i} \int \frac{d z}{(1-z)^{2}}\left(1-\rho^{n}(z)\right),
$$

where the integral goes round the unit circle. Now take $\rho(z)=(z+1)^{2} /(4 z)$, corresponding to the random variable $\frac{1}{2} B_{2}$, and substitute

$$
z=\frac{q}{w}-q-1
$$

in (2.19). We find that for all $q$ from $[1,3]$, the random variable with characteristic function

$$
\frac{1}{4}\left(q e^{-i a t}+3-q+\frac{1}{q} e^{i a t}\left(1-\theta e^{i a t}\right)^{-1}\right)
$$

has the same sum-moments as $B_{2}$, provided we take $a=2 / q, \theta=(q-1) / q .(2.21)$ is the characteristic function of $B_{2}$ when $q=1$ and is that of $G-1$ when $q=2$. 
The substitution (2.20) fails to provide a distribution whose sum-moments match those of $B_{3}$. It is still not clear to us whether $B_{3}$ is uniquely determined by its sum-moments.

We have seen that $N(0,1)$ is determined by its pseudomoments in the class of symmetric distributions but what if symmetry is dropped? It would seem to one of us that the class of all distributions is rich enough to include one with $\mathbf{E}\left|S_{n}\right|=$ $\sqrt{2 n / \pi}, n \geqq 1$. Of course such an unsymmetric distribution must have zero mean and unit variance by [EJ], but it is still a wide class. On the other hand, the same intuition would indicate that there would be many symmetric distributions with the same pseudomoments and this is not so, as we have seen. It appears to be a delicate question; the problem is that the representation of $\mathbf{E}\left|S_{n}\right|$ given in (2.3) appears not to be useful in the complex case, especially because in the form (2.5) the measure $H_{\varphi}$ is not even determined.

Remark. Numerical calculation shows that there is no distribution with support on $\{(j-2) h, j=1,2, \ldots\}$ for any $h$ with the same first 13 sum-moments as $N(0,1)$.

There is at least one distribution which is determined in general (even among nonsymmetric distributions). We know of only this one case and the proof does not use (2.3) at all. This case is the Bernoulli distribution with one degree of freedom: $\mathbf{P}\{X= \pm 1\}=\frac{1}{2}$. Note we have seen in (1.2) that if there are two degrees of freedom (and hence for any even number of degrees of freedom) then the distribution is not determined - the cases of $3,5, \ldots$, degrees of freedom remain open although it may be shown using the method of Theorem 2.1 that these laws are each unique among symmetric laws.

If $\mathbf{P}\left\{X_{k}= \pm 1\right\}=\frac{1}{2}, k \geqq 1$, and $S_{n}=X_{1}+\cdots+X_{n}$, then it is easy to verify that for $n \geqq 1$,

$$
\mathbf{E}\left|S_{2 n}\right|=\mathbf{E}\left|S_{2 n-1}\right|
$$

because $\mathbf{P}\left\{S_{2 n-1}=0\right\}=0$. But if $\left\{Y_{k}\right\}$ are i.i.d. r.v.'s which satisfy (2.22) even for $n=1$, then it follows from Jensen's inequality that there are no pair of values $a, b$ in the support of the common distribution for which $a$ and $a+b$ are nonzero and of opposite sign. Since $Y_{k}$ must have zero mean and unit variance because of the asymptotics of $\mathbf{E}\left|S_{n}\right|$, it follows easily that $Y$ must be standard Bernoulli.

The previous argument can be extended if one allow unsymmetric laws and excludes the "trivial" nonuniqueness, $X \rightarrow-X$. There is then at least one family of distributions which is determined in general (even among nonsymmetric distributions). This family is the two-point mean zero laws, which we will take to be the centered Bernoulli distribution $X=B_{q}-q$ mentioned in $\S 1$. We have the explicit formula [5]

$$
\mathbf{E}\left|S_{n}\right|=2(n q+\zeta)\left(\begin{array}{c}
n \\
n q+\zeta
\end{array}\right) q^{n q+\zeta}(1-q)^{n-n q-\zeta+1}
$$

where $n q+\zeta$ is the smallest integer larger than $n q$. It follows that if $q$ is rational, $q=a / b$ with $(a, b)=1$,

$$
\mathbf{E}\left|S_{b-1}\right|=\mathbf{E}\left|S_{b}\right|
$$

Now suppose the smallest interval containing the support of $X$ is $(f, g)$, where necessarily $f<0<g$. Then to achieve $(2.24)$ it is necessary that

$$
\mathbf{P}\left\{-g<S_{b-1}<-f\right\}=0 .
$$


Suppose the support of $X$ includes some point $h, f<h<g$. Then (by an easy induction) each of the gaps (if any) in the support of $S_{b-1}$ is at most $\max (g-h, h-f)$, which is $<g-f$, so $(2.25)$ cannot happen. If $q$ is irrational a more delicate argument is needed. By $\left[4\right.$, p. 155] we can define a constant $c_{1}$ and a sequence $n_{1}, n_{2}, \ldots$, such that $\zeta_{k}=\left\lceil n_{k} q\right\rceil-n_{k} q<c_{1} n_{k}^{-1}$. By $(2.22)$ we have

$$
\mathbf{E}\left|S_{n_{k}}\right|-\mathbf{E}\left|S_{n_{k}-1}\right|<c_{2} \zeta_{k} n_{k}^{-1 / 2}=c_{3} n_{k}^{-3 / 2} .
$$

We observe by [2] that $\sigma^{2}=\operatorname{Var} X<\infty$ is finite, since by (2.22) $\mathbf{E}\left|S_{n}\right|=O\left(n^{-1 / 2}\right)$. Now we appeal to a pair of theorems of Shepp [13], according to which if the support of $X$ is nonlattice, then

$$
\mathbf{P}\left\{-g \leqq S_{n_{k}} \leqq-f\right\} \sim(g-f)\left(2 \pi n_{k} \sigma^{2}\right)^{-1 / 2},
$$

and if the support is lattice, then in (2.27) the factor $g-f$ must be replaced by the number of support-points in $(f, g)$. In both cases we have

$$
\mathbf{E}\left|S_{n_{k}}\right|-\mathbf{E}\left|S_{n_{k}-1}\right| \geqq \mathbf{P}\left\{-g \leqq S_{n_{k}-1}<-f\right\} \min _{-g<x<-f} \mathbf{E}|x+X|,
$$

which is of the wrong order, unless $S_{n_{k}-1}$ is lattice with no support in $(-g,-f)$, which forces $X$ to have only two points of support.

3. The case $p \neq 1$. In this section all distributions considered are supposed to be symmetric. Turning to this case we face some difficulties because the analogue of the formula (2.1) is more bulky if $p \neq 1$. Let us recall that if $p$ is different from an even integer, then $p=2 m+q$, where $m$ is a nonnegative integer and $0<q<2$. Let $\vartheta(x)=\operatorname{Re} \varphi(x)$, where, as above, $\varphi(x)$ is the characteristic function of a random variable. Then

$$
\mathbf{E}|X|^{p}=c(p) \int_{0}^{\infty}\left(\sum_{j=0}^{m} \frac{(-1)^{j+1} \vartheta^{(2 j)}(0) t^{2 j}}{(2 j) !}-\vartheta(t)\right) t^{-p-1} d t
$$

where $c(p)$ is a constant (see [7]). The following statement holds $[1, \S 6]$. Suppose $\varphi$ is real and put

$$
H_{\varphi, p}(A)=\operatorname{Leb}\left\{0<u<\infty: \varphi\left(u^{-1 / p}\right) \in A\right\},
$$

where, as above, $A \subset[-1,1]$.

LEMMA 3.1. Let $p$ be different from an even integer. Suppose $\varphi$ and $\psi$ are real characteristic functions and (1.1) holds for the corresponding laws. Let $X_{k}$ have finite second moment (this assumption is nontrivial for $p<2$ only). Then $H_{\varphi, p}=H_{\psi, p}$.

Suppose $\psi$ is periodic and $2 a$ is its period. Then we may calculate $H_{\psi, p}([-1, y])$. Indeed, since $\psi(t)$ is even, then $\psi(t+a)=\psi(-t-a)=\psi(-t+a)$. So, denoting $s=\operatorname{Leb}\{t \in(0, a): \psi(t)<y\}$, we get

$$
\begin{aligned}
\text { Leb } & \left\{u>0: \psi\left(u^{-1 / p}\right)<y\right\} \\
& =\sum_{k=0}^{\infty} \operatorname{Leb}\left\{u>0:(2 k+1) a-s<u^{-1 / p}<(2 k+1) a+s\right\} \\
& =\sum_{k=0}^{\infty}\left(((2 k+1) a-s)^{-p}-((2 k+1) a+s)^{-p}\right) \equiv \chi_{a, p}(s) .
\end{aligned}
$$


Suppose there is an interval $(0, c)$, where $c \leqq a$, such that

(1) $\psi(t)$ is decreasing on $(0, c)$,

(2) $\psi(c) \geqq \sup \{\psi(t): c \leqq t \leqq a\}$.

Then the conditions $\psi(c)<y<1$ and $a-c<s<a$ are equivalent and we have

$$
\psi(a-s)=y,
$$

if $a-c<s<a$.

Let $\varphi(t)$ be real and decreasing on $(0, \infty)$. Denoting $v=H_{\varphi, p}([-1, y])$, we get $\varphi\left(v^{-1 / p}\right)=y$. From here and the previous statement the next lemma follows.

LEMMA 3.2. Let $\varphi$ and $\psi$ be real characteristic functions. Suppose $\psi$ is periodic with the period $2 a$ and there is a positive $c \leqq a$ such that the conditions (1) and (2) hold. Suppose $\varphi$ is decreasing on $(0, \infty)$. Then the relation

$$
H_{\varphi, p}([-1, y])=H_{\psi, p}([-1, y]) \quad(\psi(c) \leqq y<1)
$$

is equivalent to

$$
\psi(t)=\varphi\left(\chi_{a, p}^{-1 / p}(a-t)\right) \quad(0<t<c)
$$

Proof. Indeed, from (3.2)-(3.4) $\psi(a-s)=y=\varphi\left(\chi_{a, p}^{-1 / p}(s)\right)$ for $a-c<s<a$. Putting $t=a-s$, we get (3.5), which completes the proof.

One may easy verify that $\chi_{a, 1}(s)=\pi /(2 a) \tan (\pi s /(2 a))(0<s<a)$, which yields

$$
\chi_{a, 1}^{-1}(a-s)=\frac{2 a}{\pi} \tan \left(\frac{\pi}{2 a} s\right) .
$$

So, if $p=1$, then (3.5) gives (2.6) with $b=2 a / \pi$.

The following lemma plays the main role in the sequel. Put

$$
m=\left[\frac{p+3}{2}\right]
$$

where $[x]$ is the integer part of real $x$.

Lемма 3.3. Suppose $\varphi$ is $2 m+2$ times differentiable, $p$ is not an integer, (3.5) holds, and one of the following conditions holds:

(1) $4 k-1<p<4 k+1 \quad(k=0,1, \ldots)$;

(2) $4 k+1<p<4 k+3 \quad(k=0,1, \ldots)$ and $\psi$ is $4 k+6$-times differentiable.

Then the periodic function $\psi$ is not positive definite for any a.

Proof. The proof is based on the investigation of the asymptotic behavior of $\psi$ near zero. Denoting

$$
\theta_{a, p}(t)=\sum_{k=1}^{\infty}\left((2 k a-t)^{-p}-(2 k a+t)^{-p}\right),
$$

we obtain from $(3.3), \chi_{a, p}(a-t)=t^{-p}-\theta_{a, p}(t)$. From this and (3.5)

$$
\psi(t)=\varphi\left(t\left(1-t^{p} \theta_{a, p}(t)\right)^{-1 / p}\right) .
$$

It follows from (3.7) that $\theta_{a, p}(0)=0$ and

$$
\theta_{a, p}^{\prime}(0)=2 p a^{-p-1} \sum_{k=1}^{\infty} k^{-p-1} .
$$


So,

$$
\left(1-t^{p} \theta_{a, p}(t)\right)^{-1 / p}=1+c(a, p) t^{p+1}+o\left(t^{p+1}\right)
$$

as $t \rightarrow 0$, where $c(a, p)=2 \theta_{a, p}^{\prime}(0) / p$. Since $\varphi$ is $2 m$-times differentiable, then

$$
\varphi(t)=1+\sum_{j=1}^{m+1}(-1)^{j} c_{j} t^{2 j}+o\left(t^{2 m+2}\right)
$$

where $c_{j}$ is the moment of the degree $2 j$ divided by $(2 j)$ ! Since $p+3<2 m+2$, we get from $(3.8),(3.10)$, and (3.11)

$$
\psi(t)=1+\sum_{j=1}^{m}(-1)^{j} c_{j} t^{2 j}-2 c_{2} c(a, p) t^{p+3}+o\left(t^{p+3}\right)
$$

as $x \rightarrow+0$.

Let condition (1) hold and $p<1$. Then $m=1$ and we get from $(3.12) \psi(t)=$ $1-c_{2} t^{2}-2 c_{2} c(a, p) t^{p+3}+o\left(t^{p+3}\right)$. If $\psi$ is a characteristic function, then $n^{2}(1-\psi(t)) \geqq$ $1-\psi(n t)$ for all real $t$ and integer $n$ [9]. From here and the above one may easy derive that $c(a, p) \geqq c(a, p) n^{p+1}$ for all $n \in \mathbf{N}$, which contradicts $c(a, p)>0$.

Suppose $p>1$ and (1) holds again. Then $m=2 k+1$ and (3.12) implies $\psi$ is $2 m$-times differentiable at zero. If $\psi$ is a characteristic function, then so is $\psi^{(2 m)}(t) / \psi^{(2 m)}(0)$. But the latter is $1-b t^{2}-c t^{p+3-2 m}+o\left(t^{p+3-2 m}\right)$ as $t \rightarrow+0$, where $b$ and $c$ are positive constants, and the above leads to the contradiction.

In case (2) we have $4 k+6=2 m+2>p+3$. Since $\psi$ is supposed to be $2 m+2$ times differentiable and $p$ is not an integer we get a contradiction to (3.12) and the proof is complete.

Remark. Let us clarify why $p$ has to satisfy condition (1). Suppose $1<p<3$. Then $\psi(t)=1-t^{2} / 2+b t^{4}-c t^{p+3}+o\left(t^{p+3}\right)$ as $t \rightarrow 0$ and the reasoning fails. We don't know whether or not the lemma is true in case (2) without additional assumption on $\psi$.

We may also extend Theorem 2.1 for $p \neq 1$. Denote by $\mathcal{A}_{p}$ the class of all symmetric distributions with finite $p$ th moment if condition (1) of Lemma 3.3 holds and with finite $(4 k+6)$ th moment if $(2)$ holds.

THEOREM 3.1. If $p$ is different from an integer and $\varphi$ is analytic near zero and decreases to zero an infinity roughly smooth so that $H_{\varphi}$ has continuous Radon-Nicodym derivative on $(0,1)$ then the law of $\varphi$ is determined among the class $\mathcal{A}_{p}$.

Proof. Using Lemma 3.1 and the reasons of the proof of Theorem 2.1 we reduce the proof to the case of the periodic $\psi$. Lemmas 3.2 and 3.3 complete the proof.

Remark. In some sense the conditions of Theorem 3.1 are weaker than those of Theorem 2.1. Indeed, here we don't need to assume that $\psi$ is not positive definite for any $a$. Example (1.2) shows that the last assumption is essential for $p=1$.

Now let us turn to the case when $p$ is an odd integer. In this case the function $\chi^{-1 / p}(a-t)$ is analytic near zero and tends to infinity as $t \rightarrow a$. So, we may use the reasoning of $\S 2$ and conclude that the standard normal law is determined among the class of all symmetric distributions with finite absolute $p$ th moment. The same is true for the laws with the densities $c \exp \left(-|z|^{\alpha}\right), 1<\alpha<2$. We expect that for all odd integer $p$ there is an example of the type (1.2), but we cannot find one. 
Acknowledgments. We have benefited from remarks made by colleagues which have been incorporated into the text without explicit attribution. These colleagues are Leopold Flatto, Lev Klebanov, and Vladimir Zolotarev.

We want to express our acknowledgement to Ildar Ibragimov for the question he stated after our work was finished. He asked if it was possible for two random variables to have identically sequences of absolute sum-moment, and each of them satisfy the Cramer condition. It is doubtful that the answer is positive, although we can give only numerical reasoning. We think there exists a symmetric random variable with decreasing (for positive values of argument) characteristic function, which has the same absolute sum-moments as $e-1$, where $e$ is an exponentially distributed random variable. The proof consists of the fact that we can write the formula for the characteristic function with these absolute sum-moments (which were explicitly represented in our paper), and numerical calculations show that the characteristic functions are positive definite, i.e., the Fourier transform is definite. It seems to us that it is impossible to construct an example of this type with two symmetric random variables, but we have no proof. If it is true then it confirms that unsymmetrical random variables give more freedom for reproduction given sequence of sum-moments.

\section{REFERENCES}

[1] M. Braverman, A characterization of probability distributions by moments of sums of independent random variables, J. Theor. Prob., 7 (1994), pp. 187-198.

[2] K.-G. EsSEEN AND S. JANSON, On moment conditions for normed sums of independent random variables and martingale differences, Stoch. Proc. Appl., 9 (1985), pp. 173-185.

[3] J. Galambosh and S. Kotz, Characterizations of Probability Distributions, Lecture Notes Mathematics 675, Springer-Verlag, Berlin, 1978.

[4] G. H. Hardy and E. M. Wright, An Introduction to the Theory of Numbers, 3rd ed., Clarendon Press, Oxford, 1954.

[5] N. L. Johnson, A note on the mean deviation of the binomial distribution, Biometrika, 44 (1957), pp. 532-533.

[6] A. M. Kagan, Yu. V. Linnik, And C. R. RAO, Characterization Problems in Mathematical Statistics, Wiley, New York, 1973.

[7] T. Kawata, Fourier Analysis in Probability Theory, Academic Press, New York, London, 1972.

[8] L. B. Klebanov, A. V. Kakosian, and J. A. Melamed, Characterization of Distributions by the Method of Intensively Monotone Operators, Lecture Notes in Mathematics, 1088, Springer-Verlag, Berlin, 1984.

[9] M. LóEve, Probability Theory, 2nd rev. ed., Van Nostrand, Princeton, NJ, 1960.

[10] E. LuKACS, Characteristic Functions, 2nd ed., Griffin, London, 1970.

[11] C. L. MALlows, Bounds on the distribution function in terms of expectations of order statistics, Ann. Prob., 2 (1973), pp. 297-303.

[12] M. Neupokoeva, On the reconstruction of distributions by moments of sums of independent random variables, Stability Problems for Stochastic Models, VMIISI, Moscow, 1989, pp. 1117. (In Russian.)

[13] L. A. Shepp, A local limit theorem, Ann. Math. Stat., 35 (1964), pp. 419-423. 